\title{
Intra-operative contrast enhanced ultrasound as an adjunct to detect persistent type two endoleak after endovascular repair of abdominal aortic aneurysms
}

\author{
Regent Lee, Ediri Sideso \\ Department of Vascular Surgery, Oxford University Hospitals, NHS Foundation Trust, Oxford, UK \\ Correspondence to: Regent Lee. Department of Vascular Surgery, Oxford University Hospitals, NHS Foundation Trust, Oxford, UK. \\ Email: Regent.lee@nds.ox.ac.uk. \\ Provenance: This is an invited article commissioned by the Academic Editor Dr. Zhongzhi Jia (Department of Interventional and Vascular Surgery, \\ The Affiliated Changzhou No. 2 People's Hospital of Nanjing Medical University, Changzhou, China). \\ Comment on: Bianchini Massoni C, Perini P, Fanelli M, et al. Intraoperative contrast-enhanced ultrasound for early diagnosis of endoleaks during \\ endovascular abdominal aortic aneurysm repair. J Vasc Surg 2019. [Epub ahead of print].
}

Submitted Oct 28, 2019. Accepted for publication Nov 07, 2019.

doi: $10.21037 / \mathrm{atm} .2019 .11 .47$

View this article at: http://dx.doi.org/10.21037/atm.2019.11.47

Endovascular stent grafting (EVAR) is an established treatment of abdominal aortic aneurysms. However, a major pitfall that undermines the long-term efficacy of EVAR is persistent type II endoleaks (T2E). Previously thought to be a benign phenomenon, emerging evidence from large registries suggest aneurysm sac non-regression/expansion, likely due to persistent endoleaks, to be associated with higher cardiovascular mortality and morbidity after treatment with EVAR $(1,2)$. Novel methods to predict T2E after EVAR has potential clinical implications. Those prone to persistent T2E may be stratified towards more aggressive intervention, or, in select cases, be considered open surgical repair (OSR) instead.

In the recent manuscript presented by Massoni et al. (3), the authors described a new approach to enable early diagnosis of T2E after EVAR by performing intra-operative contrast enhanced ultrasound (CEUS). They first assessed the likelihood of persistent T2E based on morphological features documented by the pre-operative computerised tomography angiogram (CTA). High risk of persistent $\mathrm{T} 2 \mathrm{E}$ was defined as either or both (I) patency of $\geq 6$ visceral vessels afferent to the AAA, (II) the presence of a thrombus volume $<40 \%$ of the total volume of the AAA.

Over a period of 15 months, they examined the use of intraoperative CEUS during EVAR in 61 consecutive patients as an adjunct to digital subtraction angiography (DSA) to diagnose T2E. Intra-operative CEUS was successfully obtained in 60 of these patients. Where a T2E was detected base on intraoperative CEUS, additional coil embolisation of the aneurysm was performed if the patient was deemed high risk based on the pre-op CTA. Using this approach, intra-operative CEUS identified 4 cases the met the criteria of high risk for persistent $\mathrm{T} 2 \mathrm{E}$ and were embolised pre-emptively.

In this manuscript, the authors chose the agreement between DSA and intra-operative CEUS as the primary comparison. They found a very modest agreement between the two diagnostic modalities (Cohen Kappa $=0.25$ ) to detect any type of endoleak. This is an interesting choice of primary end point. One could argue that the more meaningful endpoint is whether persistent T2E is indeed eliminated in this cohort. The authors acknowledged this as a shortcoming of the current manuscript due to the short length of follow-up. The true efficacy of routine intraoperative CEUS to detect and intervene on those with high risk for persistent $\mathrm{T} 2 \mathrm{E}$ can only be assessed in a randomised controlled trial setting.

The intraluminal thrombus (ILT) within abdominal aortic aneurysms has previously been held as a bystander to the disease (4). However, emerging evidence indicates ILT to be biologically active and contains inflammatory cells within a network of canaliculi (5-7). In addition to low ILT volume as a feature of likely persistent endoleak (adopted by the Massoni et al.), it has also been observed the spatial 
distribution of ILT predicts the likelihood of T2E after EVAR. ILTs that occupy the AAA lumen circumferentially or in the postero-lateral aspect of the AAA lumen are associated with lower likelihood of T2E as compared to ILTs occupying in the other spatial distribution within the AAA lumen (8). The utility of intra-operative CEUS for pre-emptively treating T2Es could be further enhanced by taking into account these emerging novel evidence.

\section{Acknowledgments}

Funding: R Lee acknowledges the grant support from Academy of Medical Sciences, UK (AMS_SGL013\1015).

\section{Footnote}

Conflicts of Interest: The authors have no conflicts of interest to declare.

Etbical Statement: The authors are accountable for all aspects of the work in ensuring that questions related to the accuracy or integrity of any part of the work are appropriately investigated and resolved.

\section{References}

1. Deery SE, Ergul EA, Schermerhorn ML, et al. Aneurysm sac expansion is independently associated with late

Cite this article as: Lee $\mathrm{R}$, Sideso E. Intra-operative contrast enhanced ultrasound as an adjunct to detect persistent type two endoleak after endovascular repair of abdominal aortic aneurysms. Ann Transl Med 2019;7(Suppl 8):S290. doi: 10.21037/atm.2019.11.47 mortality in patients treated with endovascular aneurysm repair. J Vasc Surg 2018;67:157-64.

2. O'Donnell TFX, Deery SE, Boitano LT, et al. Aneurysm sac failure to regress after endovascular aneurysm repair is associated with lower long-term survival. J Vasc Surg 2019;69:414-22.

3. Bianchini Massoni C, Perini P, Fanelli M, et al. Intraoperative contrast-enhanced ultrasound for early diagnosis of endoleaks during endovascular abdominal aortic aneurysm repair. J Vasc Surg 2019. [Epub ahead of print].

4. Yoshimura K, Ikeda Y, Aoki H. Innocent bystander? Intraluminal thrombus in abdominal aortic aneurysm. Atherosclerosis 2011;218:285-6.

5. Folkesson M, Silveira A, Eriksson P, et al. Protease activity in the multi-layered intra-luminal thrombus of abdominal aortic aneurysms. Atherosclerosis 2011;218:294-9.

6. Rao J, Brown BN, Weinbaum JS, et al. Distinct macrophage phenotype and collagen organization within the intraluminal thrombus of abdominal aortic aneurysm. J Vasc Surg 2015;62:585-93.

7. Cassimjee I, Lee R, Jyoti P. Inflammatory Mediators in Abdominal Aortic Aneurysms. Available online: https://www.intechopen.com/books/aortic-aneurysm/ inflammatory-mediators-in-abdominal-aortic-aneurysms

8. Whaley ZL, Cassimjee I, Novak Z, et al. The Spatial Morphology of Intraluminal Thrombus Influences Type II Endoleak After Endovascular Repair of Abdominal Aortic Aneurysms. Ann Vasc Surg 2019. [Epub ahead of print]. 